\title{
Children's information retrieval: beyond examining search strategies and interfaces
}

\author{
Hanna Jochmann-Mannak \\ Human Media Interaction, Faculty of Electrical Engineering, Mathematics and Computer Science, University of Twente, \\ PO Box 217, 7500 AE Enschede, The Netherlands \\ h.e.mannak@utwente.nl \\ Theo Huibers \\ Human Media Interaction, Faculty of Electrical Engineering, Mathematics and Computer Science, University of Twente, \\ PO Box 217, 7500 AE Enschede, The Netherlands \\ t.huibers@utwente.nl \\ Ted Sanders \\ Utrecht Institute of Linguistics UiL OTS, Utrecht University, \\ Trans 10, 3512 JK Utrecht, The Netherlands \\ ted.sanders@let.uu.nl
}

\begin{abstract}
The study of children's information retrieval is still for the greater part untouched territory. Meanwhile, children can become lost in the digital information world, because they are confronted with search interfaces, both designed by and for adults. Most current research on children's information retrieval focuses on examining children's search performance on existing search interfaces to determine what kind of interfaces are suitable for children's search behaviour. However, to discover the true nature of children's search behaviour, we state that research has to go beyond examining search strategies used with existing search interfaces by examining children's cognitive processes during information-seeking. A paradigm of children's information retrieval should provide an overview of all the components beyond search interfaces and search strategies that are part of children's information retrieval process. Better understanding of the nature of children's search behaviour can help adults design interfaces and information retrieval systems that both support children's natural search strategies and help them find their way in the digital information world.
\end{abstract}

Information retrieval, search strategies, search interface, information need, conceptualizing, querying.

\section{INTRODUCTION}

Children's access to the information world is increasingly shifting from the physical library or classroom to the digital world. Every day, more children have access to the internet. Since most children nowadays grow up using computers, they seem to manage working with them better than the average adult. However, can they find relevant information in this giant information world as easily as we all might think they can? Most studies on web usability are focused on adult information-seekers. These studies report all kind of problems adults experience during information-seeking and they offer guidelines how to design user-friendly websites. The study reported in this paper focuses on children's information-seeking and discusses children's search strategies and problems, and research directions to examine how to support children's search behaviour in digital environments.

Most of the information-seeking problems experienced by children are due to the fact that search interfaces are designed by adults. Therefore, design tends to be based on adult search experiences. Unsurprisingly, search strategies required to find information are also based on adults' experience. This causes problems for children, because children are different from adults in many ways: they have other needs than adults and their cognitive, social, physical and emotional development has not yet reached the adolescent formal operational stage of development (Piaget and Inhelder, 1969, in Cooper, 2005). The most obvious differences between children and adults in information-seeking behaviour, relate to interaction style (e.g. children scroll less than adults), navigation style (e.g. adult navigation style is more systematic than child navigation style), relevance (e.g. children use different relevance criteria than adults) and mind set (e.g. children have different concepts and categories in mind than adults). To help children in effective and efficient information-seeking, it is important to know how to give them access to the information world in ways consistent with their learning, cognitive development and curriculum. 
The Netherlands Public Library Association (VOB) is aware of the importance of research on children's access to the digital information world. That is why the VOB started a research program to investigate children's search behaviour called 'The digital youth library'. The study reported in this paper is an initial exploration in the domain of children's information retrieval and the study is focused on children of 10 through 12 years which are not yet mature in their use of the internet.

Research on children's information retrieval mostly focuses on testing children's performance and examining their search strategies on a given interface. If a child's performance is, for example, more effective and efficient with a particular search tool than with another search tool, researchers may conclude that this search tool is suitable for children's search behaviour. However, to examine children's natural search behaviour, we believe that research beyond interface is needed by examining 'the black box' of children's information-seeking. That means we have to examine children's cognitive processes when they are searching for information and determine what kind of concepts and categories they have in mind, such as images, shapes, feelings, or genres. It is also important to examine at what level of abstraction children develop concepts.

As a basis for our research, we present a paradigm of children's information retrieval in Section 2, consisting of the components that model the process of a child searching for information after it has been given a particular search task. Search strategies and search interfaces are two important components of this paradigm, but also other components will be described that might even be more important in research on children's information retrieval, such as children's conceptualization and query matching with children's queries.

Section 3 discusses current research on children's search behaviour in more detail. We will present research methods and research findings concerning different search strategies and interfaces. Also found difficulties that children come across during information-seeking will be reviewed. Finally, we will discuss that current research on children's information retrieval does not expose the most important problems children encounter during informationseeking. We will discuss what kind of research we believe is needed to discover the nature of children's search behaviour and the real information-seeking problems children cope with, concerning conceptualization and query formulation.

\section{A CHILDREN'S INFORMATION RETRIEVAL PARADIGM}

The domain of children's information retrieval is not limited to searching or browsing on existing search interfaces. First, the child must have a particular search task to formulate a query, for example: what kind of food do most small birds eat? Next, the child has to conceptualize this information need, for example, by displaying an image of a sparrow in his head. After formulating a query, e.g. 'bird food', and feeding this query in an educational website for children, an information retrieval system will try to match this query with relevant documents in the information world.

In the following section every component of the paradigm, as displayed in Figure 1, will be discussed in terms of possible variants and the different effects that variants of a component can have on other components. For example, children of different ages will have different information needs and a child faced with an assigned, factdriven search task will adopt a different search strategy than a child working on a self-directed search task. All these different variants can be subject to research on children's information retrieval, so as to achieve a better understanding of the nature of children's search behaviour.

FIGURE 1: A CHILDREN'S INFORMATION RETRIEVAL PARADIGM

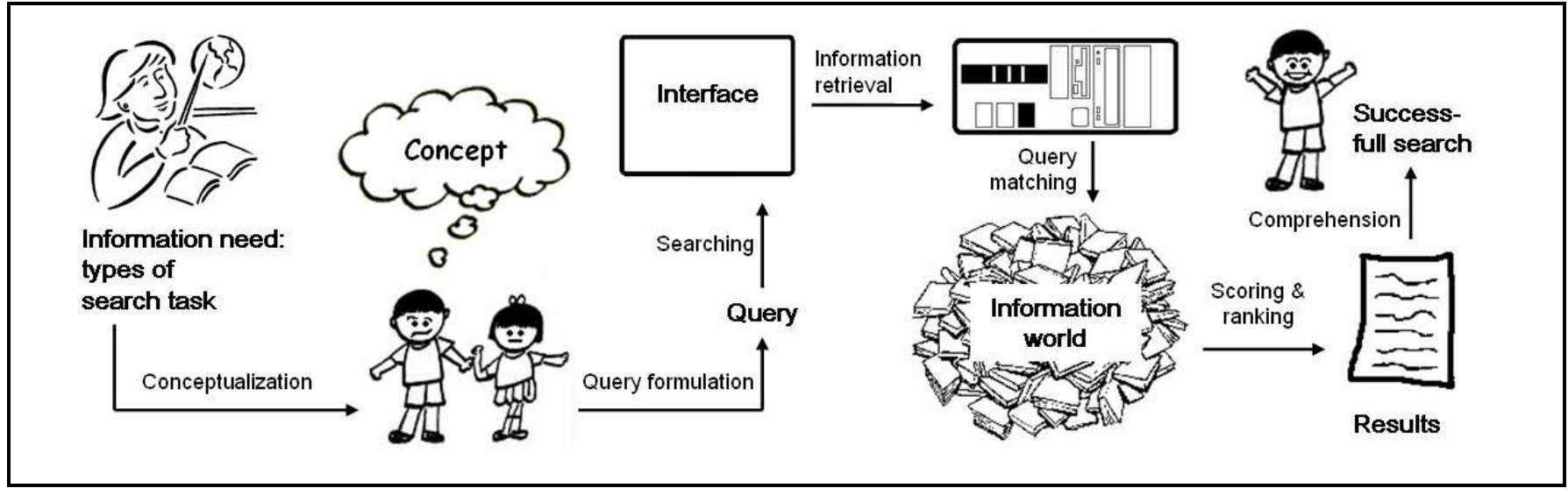




\subsection{Who are 'they' and what modulations are there?}

Children's information retrieval is not just about a child searching for information. First of all, 'a child' is a very broad term. What 'groups' of children are we talking about and do the components in the information retrieval paradigm change by different characteristics such as age, gender, reading skills, computer experience and cognitive developmental stages? To compare different 'groups' of children, it is important to group children with the same characteristics, so that found effects can be associated with the differences in that particular characteristic.

\subsection{What do 'they' want?}

What information are children looking for? In other words, what is a child's information need? What kinds of question do they have? Are these questions mostly self-directed, or externally imposed? Are the questions factbased or research-based? What is their goal for a search task: to explore, to learn, or to be entertained? Does their information need differ a lot from adults' information need? What do differences in their information needs mean for the way they formulate their information needs in a query or for the way in which they approach a search interface? The effect of change in information need on search performance can be examined by comparing different types of search task in information retrieval experiments. For example, a search task is imposed by a teacher in Figure 1.

Some research has already been conducted on examining search processes and search performance from children performing different kind of search tasks. Schacter et al. (1998) compared children's information-seeking on the internet on two tasks: well-defined tasks and ill-defined tasks. Thirty-two children in the age of 10 to 12 years participated in this experiment. The well-defined task was a closed task (i.e. fact-driven) and had a clearly defined goal in which the information necessary to solve the task was specified in the statement of the task. The ill-defined task was open ended (i.e. research-based): it had vague goals, a large number of open constraints requiring resolution, many possible solutions, and no clear directions for when to stop solving the problem. The researchers found that the children searched more effectively on the ill-defined task than on the well-defined one. Well-defined tasks were difficult for children, because they require highly skilled analytic searching strategies. III-defined tasks were easier, because there are more potential answers to ill-defined tasks. They concluded that open-ended and loosely defined search tasks are well suited for children's internet searching. On the other hand, for tasks that are well-defined and highly specific, the internet may not be the most efficient resource to assist children with their information need.

Bilal $(2000,2001,2002)$ examined children's use of the Yahooligans! web search engine and compared search performance on three kinds of search tasks: fact-based search tasks, research tasks and fully self-generated search tasks. She observed twenty-two children in the age of 12 to 13 years. Her findings were not in line with prior research (Schacter et al., 1998), because in her research children had more difficulty with the open ended, research task than with the closed, fact-based task. Bilal suggests that these opposite findings can be caused by the children's age differences between the two studies and she claims that more research in examining the effect of different search tasks on search performance is needed. The researcher also found that children were more successful on a fully self-generated task than on the assigned tasks. However, she states that this was due to children's satisfaction with the search results' content rather than the nature of the task itself (i.e. self-generated task).

\subsection{How do children conceptualize their information need?}

To formulate their information need in an utterance or query, first, children have to form a concrete concept of this need in their mind. That is why first of all, it is important to know what children think when they search for information. Are they aware that they have to formulate their information need in a concrete query or a search strategy? And does this query vary for different sources? In other words, does a child ask a different question to his mother than to the computer? Second, it is important to know how children think when they search for information. At what level of abstraction can they form a query? Can they reach the same levels of abstraction as adults, or can adults think in more abstract terms than children? What kind of categories or concepts do they have in mind: strict taxonomies, prototypes, or emotional categories? What is the role of colours, shapes, images or speech? Is this different from categories or concepts in adult minds? Knowing what kind of concepts and categories are in children's minds is important when we aim at designing interfaces suitable for children's search strategies. Research on concepts and categories in the human mind has for instance, been conducted by cognitive development psychologists, such as Murphy and Lassaline (1997). In a more recent study, Cooper (2005) addresses children's cognitive, physical, social and emotional development that has an impact on a child's ability to interact successfully with a digital environment. She discusses cognitive considerations for designing developmentally appropriate digital environments for young children. 


\subsection{How do children form a query; what are their strategies?}

Next, the child has to formulate his or her information need in a 'query'. A query is a command for a source or interface to find relevant information to satisfy the child's information need. What kind of search queries do they form: single concepts, multiple concepts, phrases or natural language? The term 'interface' here has a broad understanding; it can be a digital interface, but it can also be a physical interface such as a bookcase in a library or maybe even a father or mother to whom a child asks a question.

After a child knows what the query will be, he has the possibility to feed this query into a search system. What is his or her strategy and what is this strategy influenced by? Does this strategy differ from adult's search strategies? For example, do children prefer to browse by category or do they want to aim at precisely one goal by inserting a keyword search? Does this strategy change with search tasks? Does it change with designs of interface? In Section 3, research on different search strategies such as searching versus browsing will be discussed in more detail.

\subsection{What type of interfaces exist and how can information be offered through an interface to support children's search behaviour?}

Children's search strategies can be strongly influenced by the way the interface of a system is designed. For example, when an interface does not support browsing, because of category absence, the child has to perform keyword search to find relevant information. Different types of browsing tools - such as word clouds or image clouds, taxonomic search trees, text-based or image-based menus, social or graphical metaphors, simultaneous or sequential presentations (paging or scrolling), clustered versus faceted categories, or flat versus hierarchical presentations - can have different effects on search performance. Additionally, the way in which a search interface is designed in terms of page structure, and pictorial or typographical aspects, can have an effect on children's search performance.

Much research has already been conducted to compare children's search performance with different type of browsing tools or user interfaces. Hutchinson et al. (2006) for example compared a flat, broad and shallow presentation with a deep, narrow hierarchy. With a flat presentation (also termed a simultaneous menu), all items are concrete concepts at a single level. With a deep hierarchy, items are categorized under abstract concepts. The researchers found that for those simple tasks that did not require backtracking, users were faster using the hierarchy, but for more complex tasks, users were faster using flat, simultaneous menus.

Finally, the ways in which search interfaces are displayed can also differ. Search interfaces do not necessarily have to be displayed on a personal computer. Tangible solutions (Price et al., 2003; Blackwell et al., 2004) for displaying a search interface, such as digital tabletops, are another possibility to display search interfaces. The interface of digital tabletops is horizontally displayed to facilitate effective collaboration between children that are working together on the same interface. Sluis et al. (2004) designed Read-lt: a multimodal, tangible and collaborative tabletop application for children that supports learning to read in a novel way. In their research on this tangible interface with fifteen children in the age of 5 through 7 years, they found that the interface provided various strategies to support the learning process: recall, rehearsal and collaboration with another child. These strategies to support the learning process are found less using normal desktop interfaces.

The most important question is how to design an interface that best supports children's preferred searching behaviour. Ultimately, another question to be addressed is if such an interface also provides children's optimum search performance. Research that is focused on the effect of different types of search interface on search performance will be discussed in more detail in Section 3.

\subsection{How can an information retrieval system handle a query to find relevant documents from within 'the information world' that will satisfy the child's information need?}

The search interface serves as a user-friendly and accessible cover for a child to interact with, but actually the child is interacting with the system behind the interface. This search system is termed an Information Retrieval system (IR-system) and such a system runs the query to find relevant information. To find documents matching the query, the system has to index documents from within the information world. How can the system best select relevant documents for children? Can relevance rules for adults, that IR-systems use to decide if an information item is 'about' (i.e. relevant to) another information item (axioms of aboutness, Huibers and Bruza, 1994), also be applied on IR-systems for children? For example, when an IR-system selects 'mushroom soup' as a relevant result for the query 'toadstool', an adult will agree that this search result is relevant. However, a child may not see the relevance in 'mushroom soup' when he is searching for the house of a dwarf: a toad stool. Another question is how a system 
can handle a query while coping effectively with spelling errors or finding synonyms? Manning et al. (2008) give a thorough view on all the aspects of and previously conducted research on the domain of information retrieval.

\subsection{How can an IR-system present relevant documents?}

After an IR-system has run a query, it finds relevant results. It is important to examine how these results can be presented best for children: on the same page on which the child is searching (simultaneous) or on a new page (sequential). It is also important to examine which results must be presented first: the most relevant results by scoring and ranking the matching documents, or the documents that are most referred to by others. Further, how the individual results can best be presented for children is important: with or without a short summary of the found document. Another question is how the link labels of the results must be formulated to help children make the optimum choice. Finally, the differences and similarities between adult and child preferences for all these aspects have to be examined. Search performance on these different variants of result presentation must be examined to help decide what works best for children.

\subsection{What is successful search and what is relevance for the target group?}

What is 'the optimum choice', as mentioned above? What is relevant information for a child? What relevance criteria does a child have available to determine if a result is relevant? Are these criteria different from adults' relevance criteria? Can a child determine whether or not a result comes from a reliable source? Does it even bother a child if a document is relevant or not? What factors influence relevance judgements? Do they change during the progress of a search? What is 'successful search' to a child? In other words, what kind of search results will satisfy a child? Maybe some children will be satisfied with a result that is not even relevant. In the case of the 'small bird-example' a child may be satisfied with information about what bird food to hang in the garden to feed the birds, even though this is not the food birds eat in their natural environment.

In research on children's relevance criteria with ten children in the age of 10 to 11 years, Hirsh (1999) found that students were generally able to articulate their reasons for selecting relevant information. Important relevance criteria in her research were topicality, novelty, interest, clarity and completeness. She also found that relevance criteria changed over time while conducting a search task over a couple of weeks. Furthermore, the students in her research did not think to question the source of the information, the qualifications of the author, or the accuracy of the facts. She concludes that students need more instruction in how to search and navigate electronic resources, and how to judge the relevance of results to meet their information needs. Hirsh also believes that children need training in how to evaluate the authority and accuracy of the information they find.

\section{BACKGROUND: RESEARCH ON CHILDREN'S INFORMATION RETRIEVAL}

As mentioned before, most research on children's information retrieval focuses on search strategies on existing search interfaces. In this section research methods and research findings on search strategies that children use to find information will be discussed. Also various types of search interface and research reported on some of them will be discussed.

\subsection{Research methods for examining children's information retrieval}

Research methods used to examine children's search behaviour and search performance vary from quantitative methods such as online monitoring (Borgman, 1995; Druin, 2003) and recording activities in a browser, to qualitative methods such as discussions with focus groups (Borgman, 1995), interviews (Bilal, 2000, 2001, 2002; Borgman, 1995; Hirsh, 1999) online questionnaires (Druin, 2003), or observation of search sessions (Hirsh, 1999). With online monitoring web logs can be analyzed to gain insight into the total amount of visitors, both to websites and to individual web pages. Attitudes towards the search interface can emerge during discussions with focus groups and interviews with individual users.

In most experiments in which different types of browsing tools are compared, search performance is measured by recording the activities in a browser during task performance ((Bilal, 2001; Hutchinson, 2006; Revelle, 2002; Schacter et al. 1998). The recordings can be viewed at a later time to collect both quantitative data, such as search success, search time, efficiency and errors committed, and qualitative data, such as search behaviour and knowledge about navigation. Also, the user's ability to construct a search query with keyword search can be analyzed from these recordings. A disadvantage of this research method is that it does not give insight into the cognitive processes that occur during task performance. Recordings of task performance only show what users actually did, such as mouse movements, filling in a query, or clicking on a hyperlink. With the eye-tracking research method, eye movements of the user during task performance are recorded. Such eye-tracking data can give a researcher knowledge about the way in which an information-seeker processes particular elements in a digital environment (Ehmke et al., 2007; Guan et al., 2006). 


\subsection{Research findings on searching versus browsing}

How can children find relevant documents in the enormous amount of information provided by the internet, to meet their needs? There are many ways of making information more accessible. The way Google achieves this is by far the most preferred by adult internet users. Is the Google way of searching also most preferred by children using the internet? If so, does Google also provide optimum search performance when used by children? The Google search tool works on 'keyword search'. The user enters a query and Google returns relevant documents from the web.

"Browsing the web is an alternative to searching the web by means of a direct keyword search. Browsing is an interactive process of skimming over information and selecting choices. Browsing relies on recognition knowledge and skills, and requires less well-defined search objectives than does keyword searching." (Borgman et al., 1995)

An information-seeker can browse a website when there are systematic categories that can be selected by the user, such as semantic hierarchies, menus or search trees. Browsing relies on recognition. On the other hand, keyword search relies on recall; the user has to recall a certain term from memory. Recognition imposes less cognitive load than recall, because more knowledge is needed to retrieve terms from memory than simply to recognize offered terms. That is why a general assumption is made by researchers that browsing-oriented search tools, relying on recognition knowledge, are better suited to the abilities and skills of children than are keyword search tools (Borgman et al., 1995). However, in their research existing of four different experiments with thirty-two children per experiment, aged 9 through 12, Borgman et al. did not find any evidence for their hypothesis. This was due to the fact that keyword search in their experiment was made too easy for the participants by providing the children with the relevant subset of keywords known to match the database.

Hutchinson et al. (2006) found that children are capable of using both keyword search and category browsing, but generally prefer and are more successful with category browsing. The participants in their study were twelve children aged 6 through 7, twelve aged 8 through 9, and twelve aged 10 through 12, equally split between boys and girls. They explain this finding in relation to children's 'natural tendency to explore'. Young children tend not to plan out their searches, but simply react to the results they receive from the IR-system. Generally, their search strategies are not analytical and do not aim precisely at one goal. Instead, they make associations while browsing. This is a trial-and-error strategy.

By tracking the web logs of The International Children's Digital Library (ICDL), Druin (2003) found that, of 60,000 unique users between the ICDL's launch in November 2002 and September 2003, approximately $75 \%$ of the searches used category search (browsing), $15 \%$ used place search (by selecting a place using a world interface) and just over $10 \%$ of the searches used keyword search. Bilal (2000) found in her research on the use of the Yahooligans! Web Search Engine that most of the children (she observed twenty-two children in the age of 12 to 13 years) used keyword search. Only $36 \%$ of the searches were performed by browsing under subject categories. This finding may have been affected by the type of search task that was given in this research: a fact-driven query that automatically stimulated children to use keyword search instead of browsing the categories. She also found that children were chaotic in their search performance: they switched frequently between types of searching (i.e. keyword search or browsing), they often looped their keyword searches and selected hyperlinks, and they frequently backtracked. These findings suggest that children want to combine different search strategies during one search task.

Bilal and Kirby (2002) also found that children were more chaotic in their search performance than adults. In their research, they compared search behaviour between twenty-two children (aged 12 through 13) and twelve graduate students. Children made more web moves, they looped searches and hyperlinks more often, they backtracked more often, and they deviated more often from a designated target. The researchers concluded that adults adopted a "linear or systematic" browsing style whereas most children had a "loopy" style. They explain that this "loopy" style can be caused by children's lower cognitive recall, because the web imposes memory overload that reduces recall during navigation. They also found that children scrolled result pages less often than adults.

Schacter et al. (1998) found that with both highly specific and vague search tasks, children sought information by using browsing strategies. In their research on children's internet searching on complex problems with thirty-two children in the age of 10 to 12 years, they reported the following.

"Children are reactive searchers who do not systematically plan or employ elaborated analytic search strategies." 
Finally, Revelle et al. (2002) report on the development of a visual search interface to support children in their efforts to find animals in a hierarchical information structure. To examine searching and browsing behaviour, 106 children (aged 5 through 10) participated in an experiment on this visual search interface. The researchers found that:

"(...) even young children are capable of efficient and accurate searching. With the support of a visual query interface that includes scaffolding for Boolean concepts, children can use a hierarchical structure to perform searches and construct search queries that surpass their previously demonstrated abilities with the use of traditional search techniques."

\subsection{Children's problems on information-seeking}

In research on search behaviour, researchers often find that children experience difficulties while using both searching and browsing tools. These tools do not take into account children's information processing and motor skills (Hutchinson et al., 2006).

\subsubsection{Difficulties concerning motor skills}

Concerning motor skills, children can have difficulties using a mouse, because they process information more slowly than adults. The smaller the object to be clicked on, the longer it takes for a child to click on it (Fitts, 1995, in Hutchinson et al., 2005). Second, many children have difficulty with typing. They are not yet capable of typing without looking at the keyboard, termed touch-typing. Instead, they 'hunt and peck' on the keyboard for the correct keys (Borgman, 1995). That is why typing for children often takes a long time and can lead to frustration.

\subsubsection{Difficulties with searching and browsing}

Usually, formulating a search query is difficult for children, because they have little knowledge to base 'recall' on (Borgman et al., 1995; Hutchinson, 2005). Besides, for searching relevant documents using keyword search, correct spelling, spacing and punctuation are needed. Children have difficulty with spelling and often make spelling errors (Borgman et al., 1995). That is why an information retrieval system should be able to handle spelling errors, to help children find relevant documents using keyword search. Deciding on a single keyword is also difficult for a child, because children tend to use a full natural language query. Thus, a system should also be able to handle natural language queries to find relevant information. In a comparison study between children and adults, Bilal and Kirby (2002) found that when children employed keyword searching, most of their queries were single or multiple concepts, just like adults. However, adults employed advanced search syntax, while children did not use this syntax.

With category search, children first of all have trouble finding the right category, because they have little domainknowledge to decide which category is optimum. In addition, problems with browsing tools are mostly the result of a lack of vocabulary knowledge. Children often have difficulties understanding abstract, top-level headings, because their vocabulary knowledge is not yet sufficient to understand such terms (Hutchinson, 2006). Therefore, formulation of headings should be adjusted to children's vocabulary knowledge, using simple, concrete search terms. Also children may not think hierarchically like adults and may have trouble understanding the way in which hierarchically based categories are constructed. Knowing what their understanding of categories is, can therefore be of great value in designing browsing tools. Bar-llan and Belous (2007) tried to understand what browsable, hierarchical subject categories children create by conducting a cardsorting experiment with twelve groups of four children in the age of 9 through 11 years. They suggested terms to the children through 61 cards. The children were free to add, delete or change terms. The researchers found that the majority of the category names used by existing directories were acceptable for the children and only a small minority of the terms caused confusion. Finally, often information in browsing systems is alphabetically displayed, requiring good alphabet skills. Many children have problems with alphabetizing and therefore have trouble finding information in such browsing systems (Borgman, 1995).

\subsubsection{Difficulties concerning 'the black box'}

Most browsing tools do not consider how children prefer to search and use search criteria which work for adults. Children use different search criteria than adults. For example, they like to search by physical attributes such as images, colours and shapes (Hutchinson et al., 2005). Also children like to search by concrete genres such as animals or sports, or by feelings and emotions such as happy, sad, scary or sweet. It is important to know which kind of search criteria children use in designing browsing tools. However, we do not know exactly what children's search criteria are and what goes on in their minds when they search for information. That is why we call this 'the black box' of children's information retrieval. 


\section{DISCUSSION: BEYOND EXAMINING CHILDREN'S SEARCH STRATEGIES USING EXISTING SEARCH INTERFACES}

According to previous research, most of the problems children experience with searching and browsing are due to search interfaces that do not take into account both children's low motor skills and their different approaches to searching and browsing in comparison to adults. This is because most search interfaces are designed by adults and are therefore based on skills and preferences of adults.

However, does research that focuses on the interface really uncover the heart of the matter? Can we support children's information retrieval just by knowing which search tools do and do not work for them? If research reports that children perform better with a particular search tool in comparison to another search tool, can we then conclude that this search tool also provides an approach preferred by children. Does this approach align with their natural search behaviour? What is their natural search behaviour? Does it give optimum results or do more mature search strategies give better results? What factors, other than interface design, can have an influence on children's search behaviour? Do search strategies change according to the type of search task? Finally, are search strategies different for different kind of children?

To examine the nature of children's search behaviour, we have to go beyond examining performance on existing search interfaces by examining 'the black box' of children's information retrieval. For example, testing an interface using the eye-tracking method, can give insight in the way a child processes a particular search interface, but it does not say anything about the cognitive processes such as conceptualization in a child's brain while conducting the search task. How can we find out what kind of categories children would like to select, maybe based on colours, images, shapes or feelings? Cardsorting (Bar-llan and Belous, 2007), for example, is a plausible method to discover children's preferred categories. However, the disadvantage of this method is that term suggestions are given to the child in stead of formulated by the child itself. How can we find out what concepts children develop and at what level of abstraction they develop concepts in their brains? In research children should be encouraged to formulate concepts themselves. To determine children's concept levels of abstraction, children can be showed pictures and asked to name them. For example, a child can name a picture of a canary 'animal' (superordinate level), 'bird'(basic level) or 'canary' (subordinate level) (Murphy and Lassaline, 1997).

Another important question in achieving optimum search results for children concerns relevance. Can an IR-system handle children's queries in the same matter as adult queries? In other words, is a document that is relevant to an adult's query also relevant to a child's query? Can relevance rules for adults, that IR-systems use to decide if an information item is 'about' (i.e. relevant to) another information item (axioms of aboutness, Huibers and Bruza, 1994), also be applied on IR-systems for children? In research, relevance of found results of IR-systems based on adult relevance rules, should be judged by children.

We believe that research addressing these questions will give insight in the real problems children experience with information-seeking, so that we can support them in their search for information. In this research, the impact of factors such as children's motor skills, domain knowledge, searching and browsing skills, reading and writing skills, and alphabet skills must be minimized, so that found effects can only be caused by experimental factors and not by differences in these mentioned factors.

\section{CONCLUSIONS AND FUTURE RESEARCH}

Previous research showed that children have trouble finding information using adult search tools. Therefore, an important question in the area of children's information retrieval is how to design a search interface that is suitable for children. Most of the research on this subject focuses on testing children's search performance on existing search interfaces. However, we believe that such research does not address the real problems children cope with during information-seeking concerning conceptualization and query formulation.

In our research program 'The digital youth library' we will conduct experiments to discover what is in 'the black box' by examining children's cognitive processes during information-seeking. Only in that way it can be discovered what kind of search strategies children prefer and if such strategies indeed give optimum results for children, because then their strategy is not conditioned by the existing adult-based search interfaces. Better understanding of children's search behaviour will eventually help adults design interfaces and IR-systems that better support children's natural search strategies. 


\section{ACKNOWLEDGMENTS}

This study is funded by The Netherlands Public Library Association and is part of their research program called 'The digital youth library'. This program is a cooperation between several Dutch public libraries to develop a digital youth library. For the development of this youth library, input from academic research is required to design a developmentally appropriate digital environment for children.

\section{REFERENCES}

[1] Bar-Ilan, J. and Belous, Y. (2007) Children as Architects of Web Directories: An Exploratory Study. Journal of the American Society for Information Science and Technology, 58, 895-907.

[2] Bilal, D. (2000) Children's Use of the Yahooligans! Web Search Engine: I. Cognitive, Physical, and Affective Behaviors on Fact-Based Search Tasks. Journal of the American Society for Information Science and Technology, 51, 646-665.

[3] Bilal, D. (2001) Children's Use of the Yahooligans! Web Search Engine: II. Cognitive and Physical Behaviors on Research Tasks. Journal of the American Society for Information Science and Technology, 52, 118-136.

[4] Bilal, D. (2002) Children's Use of the Yahooligans! Web Search Engine: III. Cognitive and Physical Behaviors on Fully Self-Generated Search Tasks. Journal of the American Society for Information Science and Technology, 53, 1170-1183.

[5] Bilal, D. and Kirby, J. (2002) Differences and similarities in information seeking: children and adults as Web users. Information Processing and Management, 38, 649-670.

[6] Blackwell, A.F., Stringer, M., Toye, E.F. and Rode, J.A. (2004) Tangible Interface for Collaborative Information Retrieval. Proceedings of $\mathrm{CHI}$ 2004, Vienna, Austria, 24-29 April, pp. 1473-1476.

[7] Borgman, C.L., Hirsh, S.G. and Walter, V.A. (1995) Children's Searching Behavior on Browsing and Keyword Online Catalogs: The Science Library Catalog Project. Journal of the American Society for Information Science, 46, 663-684.

[8] Cooper, L.Z. (2005) Developmentally Appropriate Digital Environments for Young Children. Library Trends, 54, 286-302.

[9] Druin, A. (2005) What Children Can Teach Us: Developing Digital Libraries for Children. Library Quarterly, 75, 20-41.

[10] Ehmke, C. and Wilson, S. (2007) Identifying Web Usability Problems from Eye-Tracking Data. Proceedings of $\mathrm{HCl}$ 2007, Rio de Janeiro, Brazil, 20 October, British Computer Society.

[11] Guan, Z., Lee, S., Cuddihy, E. and Ramey, J. (2006) The Validity of the Stimulated Retrospective Think-Aloud Method as Measured by Eye Tracking. Proceedings of CHI '06, Québec, Canada, 22-27 April, pp. 1253-1262.

[12] Hirsh, S.G. (1999) Children's Relevance Criteria and Information Seeking on Electronic Resources. Journal of the American Society for Information Science, 50, 1265-1283.

[13] Huibers, T.W.C. and Bruza, P. (1994) Situations, a General Framework for Studying Information Retrieval. Proceedings of the 16th Research Colloquium of the British Computer Society Information Retrieval Specialist Group, Drymen, Schotland, March, pp. 3-24.

[14] Hutchinson, H., Bederson, B.B. and Druin, A. (2006) The Evolution of the International Children's Digital Library Searching and Browsing Interface. Proceedings of IDC '06, Tampere, Finland, 7-9 June, pp. 105-112.

[15] Hutchinson, H., Druin, A., Bederson, B.B., Reuter, K., Rose, A. and Weeks, A.C. (2005) How do I find blue books about dogs? The errors and frustrations of young digital library users. Proceedings of HCII 2005, Las Vegas, NV, 22-27 July.

[16] Manning, C.D., Raghavan, P. and Schutze, H. (2008) An Introduction to Information Retrieval. Cambridge University Press, Cambridge. (Preliminary draft, printed on January 25, 2008).

[17] Murphy, G.L. and Lassaline, M.E. (1997) Hierarchical Structure in Concepts and the Basic Level of Categorization. In Lamberts, K. and Shanks, D. (eds), Knowledge, concepts and categories. Psychology Press, London.

[18] Price, S., Rogers, Y., Scaife, M., Stanton, D. and Neale, H. (2003) Using 'tangibles' to promote novel forms of playful learning. Interacting with Computers, 15, 169-185.

[19] Revelle, G., Druin, Al, Platner, M., Bederson, B., Houcade, J.P. and Sherman, L. (2002) A Visual Search Tool for Early Elementary Science Students. Journal of Science Education and Technology, 11, 49-57.

[20] Schacter, J., Chung, G.K.W.K. and Dorr, A. (1998) Children's Internet Searching on Complex Problems: Performance and Process Analyses. Journal of the American Society for Information Science, 49, 840-849.

[21] Sluis, R.J.W., Weevers, I., van Schijndel, C.H.G.J., Kolos-Mazuryk, L., Fitrianie, S. and Martens, J.B.O.S. (2004) Read-It. Five-to-seven-year-old children learn to read in a tabletop environment. Proceedings of the IDC '04 Conference on interaction Design and Children: Building A Community, Maryland, USA, 1-3 June, pp. $\sim 73-80$. 